\title{
Legislation on violence against women: overview of key components
}

\author{
Gaby Ortiz-Barreda ${ }^{1}$ and Carmen Vives-Cases ${ }^{2}$
}

Suggested citation Ortiz-Barreda G, Vives-Cases C. Legislation on violence against women: overview of key components. Rev Panam Salud Publica. 2013;33(1):61-72.

\begin{abstract}
This study aimed to determine if legislation on violence against women (VAW) worldwide contains key components recommended by the Pan American Health Organization (PAHO) and the United Nations (UN) to help strengthen VAW prevention and provide better integrated victim protection, support, and care.

A systematic search for VAW legislation using international legal databases and other electronic sources plus data from previous research identified 124 countries/territories with some type of VAW legislation. Full legal texts were found for legislation from 104 countries/ territories. Those available in English, Portuguese, and Spanish were downloaded and compiled and the selection criteria applied (use of any of the common terms related to VAW, including intimate partner violence (IPV), and reference to at least two of six sectors (education, health, judicial system, mass media, police, and social services) with regard to VAW interventions (protection, support, and care). A final sample from 80 countries/territories was selected and analyzed for the presence of key components recommended by PAHO and the UN (reference to the term "violence against women" in the title; definitions of different types of VAW; identification of women as beneficiaries; and promotion of (reference to) the participation of multiple sectors in VAW interventions).

Few countries/territories specifically identified women as the beneficiaries of their VAW legislation, including those that labeled their legislation "domestic violence" law $(\mathrm{n}=51)$, of which only two explicitly mentioned women as complainants/survivors. Only 28 countries/ territories defined the main forms of VAW (economic, physical, psychological, and sexual) in their VAW legislation. Most highlighted the role of the judicial system, followed by that of social services and the police. Only 28 mentioned the health sector.

Despite considerable efforts worldwide to strengthen VAW legislation, most VAW laws do not incorporate the key recommended components. Significant limitations were found in the legislative content, its application, and the extent to which it provided women with integrated protection, support, and care. In developing new VAW legislation, policymakers should consider the vital role of health services.
\end{abstract}

Key words Violence against women; domestic violence; health policy; legislation; Americas; Europe; Asia; Africa; Oceania.

Violence against women (VAW) remains a crucial factor undermining women's ability to enjoy fundamental

\footnotetext{
${ }^{1}$ Grupo de Investigación de Salud Pública, Universidad de Alicante, Alicante, Spain. Send correspondence to: Gaby Ortiz Barreda, gaby.ortiz@ua.es

${ }^{2}$ CIBER de Epidemiología y Salud Pública (CIBERESP), Spain.
}

freedoms (1) and represents both a serious violation of human rights and a major obstacle to overcoming inequality between women and men in all societies $(1,2)$. Intimate partner violence (IPV) is one of the most common forms of VAW and occurs in all societies irrespective of social and economic systems, religion, and culture $(1,3)$. IPV perpetrators are almost exclusively men $(1,4)$.

In 2005, the World Health Organization (WHO) Multi-country Study on Women's Health and Domestic Violence Against Women reported that up to $50 \%$ of women had suffered physical injuries as a result of IPV, and that almost $20 \%$ 
of IPV victims had never reported the violence to any person or agency (5). IPV has a profound impact on mental and physical health (6). Empirical evidence has highlighted the "cumulative effect" of various forms of abuse, indicating that the health of women who experience a combination of physical, sexual, and psychological abuse is more severely affected than that of women who experience a single form of abuse $(6,7)$.

The cost of IPV increases the financial burden on societies by causing lost productivity as well as greater use of social services $(1,8,9)$. Based on data from the National Violence Against Women Survey (NVAWS), the U.S. Centers for Disease Control and Prevention (CDC) estimated that the direct costs of medical and mental health care services related to IPV exceeded US\$ 4.1 billion annually (8). Women subjected to this violence are at greater risk of poverty, divorce, workplace absenteeism, and unemployment (10). The negative effects of IPV on the workplace represent a cost to employers (11).

Despite the abundant literature on the various consequences of IPV on women's health and economic well-being, little research has been conducted on the legislation enacted to protect women and societies from this type of violence.

\section{United Nations efforts to promote VAW policies}

Many countries/territories worldwide have adopted VAW legislation (or revised existing legislation, by passing laws and legal codes, as well as amendments to their constitutions) to address VAW more effectively $(12,13)$. This may be a response to the significant progress spearheaded by the United Nations (UN) in developing international standards and treaties to address VAW. In 1993, the UN World Conference on Human Rights in Vienna recognized that VAW constitutes a severe violation of human rights and called for the development of international, regional, and national programs to address this type of violence as well as discrimination against women (14). During the UN Fourth World Conference on Women held in Beijing in 1995, several countries/territories adopted the Beijing Platform for Action (PFA), which stated that VAW prevents women from enjoying their human rights and fundamental freedoms (15) and called for national governments to assume full responsibility for the development and implementation of national policies to address VAW by strengthening and extending their existing legislation (15). Recognition of women's rights in the public and private sphere was also strengthened by the UN General Assembly Declaration on the Elimination of Discrimination Against Women (DEDAW) (1967) and the Convention on the Elimination of All Forms of Discrimination Against Women (CEDAW) $(1979)(16,17)$.

UN efforts have been influential in "women's-rights-as-human-rights" campaigns and in the linking of violence against women to the women's human rights movement. The human rights approach is being used by both international organizations and the women's movement to put pressure on country/ territory governments worldwide to develop laws and gender policies to protect women and to improve women's access to justice $(14,18)$. The European Women's Lobby (EWL) Observatory on VAW has already highlighted VAW legislation as an indicator of a country/territory's level of commitment and willingness to act by proposing legal mechanisms for the protection and defense of women (19).

\section{Guidelines for developing VAW legal tools}

In the last decade, international organizations such as the UN and the Pan American Health Organization (PAHO) have indicated the importance of developing model frameworks for the design, application, and evaluation of VAW laws and public policies (20-22). According to these model frameworks, legislation should be comprehensive and multidisciplinary, criminalizing the main forms of VAW (economic, psychological, physical, and sexual), and integrating interventions related to VAW prevention as well as protection, support, and care for victims. Adequate punishment of perpetrators and availability of remedies for survivors (20, 21 ), as well as educational interventions and awareness-raising activities, and professional training for those working to reduce VAW, are also recommended (20, $21,23,24)$. Therefore, the participation of multiple sectors (e.g., education, health, the judicial system, mass media, police, and social services) is required.
In 1993, the XXXVIII Directing Council of PAHO urged governments in the Americas region to adopt policies and plans for the prevention and control of violence (25). A year later, the Organization of American States (OAS) adopted the Inter-American Convention on the Prevention, Punishment and Eradication of Violence Against Women ("Convention of Belém do Pará") (1994) as a legally binding instrument for its member states (26). The legal framework established by this Convention has stimulated the development of VAW laws and policies in many countries/territories in the Americas.

In 2003, PAHO highlighted the importance of key VAW legislative components (minimum, nonnegotiable elements that all VAW laws should include) (22). One key element is the naming style used for the laws. The use of general or imprecise terms in the titles of the legislation (e.g., "intra-family violence" or "domestic violence") may weaken the judicial protection available to victims of VAW by opening it up to conditions (22). Therefore, specific reference to violence directed against women in the title of the legislation is recommended.

Other recommendations for VAW legal texts include 1) clear definitions of the main forms of VAW (20-22); 2) the specific identification of women as beneficiaries of the legislation; and 3) promotion of (reference to) the participation of multiple sectors in VAW interventions (20-22).

Although many nations have signed and ratified the UN conventions calling for the development and implementation of national VAW legislation, previous studies provide evidence that laws enacted to date are limited in terms of content and application (27-30). Furthermore, it remains unclear whether the legislation aligns with the internationally agreed-upon standards. The aim of this study was to determine if the key legislative components recommended by international organizations are present in VAW legislation worldwide. Highlighting the gaps in existing legislation with regard to internationally recommended key components may help lead to legal reforms of current laws or the creation of new laws that provide better-integrated VAW prevention and victim protection, support, and care. 


\section{MATERIALS AND METHODS}

\section{Data collection}

A systematic search for VAW legislation from countries/territories worldwide was conducted using three international legal databases (Harvard University's Annual Review of Population Law (ARPL) (31), the UN Secretary-General's Database on Violence Against Women (32), and Advocates for Human Rights "Stop Violence Against Women" (33)); other electronic sources; and data from a previous study on legal tools legislated between January 1984 and June 2012 (30). A total of 124 countries/territories had some type of legislation to address VAW. Full-text legislative documents were found for 104 countries/territories. Those available in English, Portuguese, and Spanish were downloaded and compiled and the selection criteria applied (use of any of the common terms related to VAW, including IPV, and reference to at least two of six sectors (education, health, judicial system, mass media, police, and social services) with regard to VAW interventions for victim protection, support, and care). A final sample from 80 countries/territories was selected and analyzed for the presence of key components recommended by $\mathrm{PAHO}$ and the UN (reference to the term "violence against women" in the title; definitions of different types of VAW; identification of women as beneficiaries; and promotion of (reference to) the participation of multiple sectors in VAW interventions. Countries/territories whose legal texts were limited to married women emancipation acts, legal reforms, penal codes, penal code amendments, and/ or draft legislation were excluded from the study. The final sample consisted of legislation from 29 countries/territories from the Americas, 17 from Europe, 23 from Asia, 10 from Africa, and 1 from Oceania (Table 1).

\section{Content analysis measures}

The content analysis included two sets of measures: general information variables, and key recommended legislative components. General information variables included the year in which the legislation was enacted and the geographic region of the country/territory of origin, according to the UN Secretary-General's Database on Violence against Women (32). Key recommended legislative components included the following:

- Naming style (terms used in the legislation title). Categories included "violence against women," "domestic violence," "intra-family violence," "gender violence," "family violence," and "other."

- Definitions of the main forms of VAW. Categories included "economic," "physical," "psychological," "sexual," and "other," and were not mutually exclusive.

- Identification of women as beneficiaries (i.e., eligible for the protection, support, and care provided by the legislation). Categories included "refers to women only," "refers to women along with other family members," and "refers to 'the family' only" (i.e., no explicit mention of women as complainants and/or survivors of violence).

- Promotion of (reference to) the participation of multiple sectors in VAW interventions (protection, support, and care). Categories included "education," "health," "judicial system," "mass media," "police," and "social services," and were not mutually exclusive.

\section{RESULTS}

In the Americas, 29 countries/ territories had VAW legislation that met the study inclusion criteria, the majority of which was written between the years 2000 and 2012. Puerto Rico was the first in the region to legislate on VAW, with the enaction of its Domestic Violence Prevention and Intervention Law (Ley de Prevención e Intervención con la Violencia Doméstica) in 1989. In Europe, VAW legislation from 17 countries/territories was included in the study (all written between the years 2000 and 2012). Ukraine was the first European country to enact a VAW law (Law on Prevention of Domestic Violence) (2001). In Asia, 23 countries had VAW legislation that met the study criteria (written between 1994 and 2012). Malaysia was the first to enact VAW legislation (Domestic Violence Act 1994). Africa and Oceania had fewer countries/territories with VAW legislation that met the study criteria (10 and 1 respectively). The proportion of countries in each region with VAW legislation analyzed in the study is shown by year (1984-2012) in Figure 1.

Results of the "naming style" component of the content analysis (use of specific terms in the title) revealed a total of 51 of the 80 countries/territories included the term "domestic violence" in the title of their legislation (15 from the Americas; 11 from Europe; 16 from Asia; 8 from Africa; and 1 from Oceania). The total number of countries/territories that used the other four terms in their legislative titles was lower: "violence against women" ( $n=14)$; "family violence" $(n=8)$; "intra-family violence" $(n=1)$; and "gender violence" $(n=2)$. Spain and Rwanda were the two countries that used the term "gender violence."

Examining the full legal texts revealed that most $(n=44)$ of the 51 countries/ territories that used the term "domestic violence" in their legislative titles specified "the family" as the beneficiary of the legal protection (i.e., women were not explicitly mentioned as potential complainants and/or survivors of violence). Only two countries/territories from that subgroup specified women as a target beneficiary of the legislation. On the other hand, among the 14 countries/territories that used the term "violence against women" in the title of their legislation, nine specified women as the sole beneficiary of the legal protection, and five specified women along with other family members as the target beneficiaries (Table 2).

A total of 39 countries/territories included definitions of some of the main forms of VAW in their legislation: 28 defined four types of abuse ("economic," "physical," "psychological," and "sexual"); six described three ("physical," "psychological," and "sexual"); two described two types of abuse ("physical" and "psychological"); and three defined one ("psychological"). Argentina, Brazil, Colombia, Costa Rica, El Salvador, Guatemala, Honduras, Mexico, Mozambique, Nicaragua, the Republic of Moldova, Uruguay, and Venezuela included definitions of other types of VAW ("femicide," "institutional," and "misogyny") in their legislation.

Figure 2 shows the level of participation in VAW victim interventions by sector, based on the number of countries/ territories that referred to each sector in their VAW legislation. For all geographic regions, the legislation appeared to em- 
TABLE 1. Selected legislation on violence against women (VAW) from countries/territories worldwide ( $n=80)$, by region, January $1984-J u n e ~ 2012^{a-c}$

\begin{tabular}{|c|c|c|}
\hline Region & Country/territory & Name of legislation, year of approval, and source \\
\hline \multirow[t]{21}{*}{ Americas } & $\begin{array}{l}\text { Antigua and } \\
\text { Barbuda }\end{array}$ & $\begin{array}{l}\text { Domestic Violence (Summary Proceedings) Act } 1999 \\
\text { Available from: http://sgdatabase.unwomen.org/uploads/Antigua\%20and\%20Barbuda\%20-\%20Domestic\%20Violence\%20-\%20 } \\
\text { Summary\%20Proceedings\%20-\%20Act\%201999.pdf }\end{array}$ \\
\hline & Bahamas & $\begin{array}{l}\text { Domestic Violence (Protection Orders) Act, } 2007 \\
\text { Available from: http://sgdatabase.unwomen.org/uploads/The\%20Domestic\%20Violence\%20Act\%202007.pdf }\end{array}$ \\
\hline & Barbados & $\begin{array}{l}\text { Domestic Violence (Protection Orders) Act (1993) } \\
\text { Available from: http://sgdatabase.unwomen.org/uploads/Domestic\%20Violence\%20Protection\%20Orders\%20Act\%201993.pdf }\end{array}$ \\
\hline & Belize $^{d}$ & $\begin{array}{l}\text { Domestic Violence Act (revised edition 2000) } \\
\text { Available from: http://sgdatabase.unwomen.org/uploads/Belize\%20-\%20Domestic\%20Violence\%20Act\%20Chapter\%20 } \\
\text { 178\%20\%282000\%29.pdf } \\
\text { Domestic Violence Act, } 2007 \text { (reform law) } \\
\text { Available from: http://sgdatabase.unwomen.org/uploads/Domestic\%20Violence\%20Act\%202007.pdf }\end{array}$ \\
\hline & Bermuda & $\begin{array}{l}\text { Domestic Violence (Protection Orders) Act } 1997 \\
\text { Available from: http://www.hsph.harvard.edu/population/domesticviolence/bermuda.dv.97.pdf }\end{array}$ \\
\hline & Bolivia $^{d}$ & $\begin{array}{l}\text { Ley contra la Violencia en la Familia o Doméstica (No. 1674, 1995) } \\
\text { Available from: http://www.hsph.harvard.edu/population/domesticviolence/bolivia.dv.95.htm } \\
\text { Decreto Supremo № } 25087 \text { (reform of law 1674, 1998) } \\
\text { Available from: http://www.hsph.harvard.edu/population/domesticviolence/bolivia.dv.95\%282\%29.htm }\end{array}$ \\
\hline & Brazil & $\begin{array}{l}\text { Maria da Penha Law (English translation of law } 11340 \text { of 2006, a classification/definition of domestic and family violence against women) } \\
\text { Available from: http://sgdatabase.unwomen.org/uploads/Brazil\%20-\%20Maria\%20da\%20Penha\%20Law\%20\%282006\%29.pdf }\end{array}$ \\
\hline & Cayman Islands & $\begin{array}{l}\text { Summary Jurisdiction (Domestic Violence) Law, } 1992 \\
\text { Available from: http://www.hsph.harvard.edu/population/domesticviolence/CAYMANISLANDS.htm }\end{array}$ \\
\hline & Chile $^{d}$ & $\begin{array}{l}\text { Ley de Violencia Intrafamiliar (No. 20066, 2005) } \\
\text { Available from: http://sgdatabase.unwomen.org/uploads/Ley\%2020066\%20of\%202005\%20on\%20Domestic\%20Violence.pdf } \\
\text { Modifica el Código Penal y la Ley no } 20.066 \text { sobre Violencia Intrafamiliar, estableciendo el "Femicidio" aumentado las penas aplicables a } \\
\text { este delito y reforma las normas sobre parricidio (No. 20480, 2010) } \\
\text { Available from: http://sgdatabase.unwomen.org/uploads/Ley\%2020-480\%20-\%20femicidio\%20-\%202010.pdf }\end{array}$ \\
\hline & Colombia & $\begin{array}{l}\text { Ley } 1257 \text { de Diciembre } 4 \text { de } 2008 \text { ("Por la cual se dictan normas de sensibilización, prevención y sanción de formas de violencia } \\
\text { y discriminación contra las mujeres, se reforman los Códigos Penal, de Procedimiento Penal, la ley } 294 \text { de } 1996 \text { y se dictan otras } \\
\text { disposiciones"; 2008) } \\
\text { Available from: http://www.hsph.harvard.edu/population/domesticviolence/colombia.sexcrimes.08.pdf }\end{array}$ \\
\hline & Costa Rica & $\begin{array}{l}\text { Ley de Penalización de la Violencia contra la Mujer (No. 8589, 2007) } \\
\text { Available from: http://sgdatabase.unwomen.org/uploads/Costa\%20Rica\%20-\%20Ley\%20Penalizacion\%20de\%20violencia\%20 } \\
\text { contra\%20mujer.pdf }\end{array}$ \\
\hline & Ecuador & $\begin{array}{l}\text { Ley contra la Violencia a la Mujer y a la Familia (No. 103, 1995) } \\
\text { Available from: http://sgdatabase.unwomen.org/uploads/Ecuador\%20-\%20Ley\%20contra\%20la\%20violencia\%20a\%20la\%20 } \\
\text { mujer\%20y\%20la\%20familia.pdf }\end{array}$ \\
\hline & El Salvador & $\begin{array}{l}\text { Ley Especial Integral para una Vida Libre de Violencia para las Mujeres }(2010) \\
\text { Available from: http://sgdatabase.unwomen.org/uploads/Ley\%20Especial\%20Integral\%20Vida\%20Libre\%20Violencia\%20 } \\
\text { Mujeres\%20-\%202011.pdf }\end{array}$ \\
\hline & Guatemala & $\begin{array}{l}\text { Ley contra el Femicidio y otras formas de Violencia contra la Mujer (No. 22, 2008) } \\
\text { Available from: http://sgdatabase.unwomen.org/uploads/Guatemala\%20-\%20Ley\%20Femicidio.pdf }\end{array}$ \\
\hline & Guyana & $\begin{array}{l}\text { Domestic Violence Act (1996) } \\
\text { Available from: http://sgdatabase.unwomen.org/uploads/Domestic\%20Violence\%20Act.pdf }\end{array}$ \\
\hline & Honduras $^{d}$ & $\begin{array}{l}\text { Ley contra la Violencia Doméstica reformada (revised edition of law enacted in } 1997 \text { including reforms enacted in 2005) } \\
\text { Available from: http://sgdatabase.unwomen.org/uploads/Ley\%20Contra\%20la\%20Violencia\%20Domestica\%20Reformada.pdf }\end{array}$ \\
\hline & Jamaica $^{d}$ & $\begin{array}{l}\text { Domestic Violence Act (revised edition of law enacted in } 1996 \text { including reforms enacted in 2004) } \\
\text { Available from: http://sgdatabase.unwomen.org/uploads/Domestic\%20Violence\%20Act\%202004.pdf }\end{array}$ \\
\hline & Mexico ${ }^{\mathrm{d}, \mathrm{e}}$ & $\begin{array}{l}\text { Ley General de Acceso de las Mujeres a una Vida Libre de Violencia (revised edition of law enacted in } 2006 \text { including reforms enacted in 2012) } \\
\text { Available from: http://www.diputados.gob.mx/LeyesBiblio/pdf/LGAMVLV.pdf }\end{array}$ \\
\hline & Nicaragua $^{d}$ & $\begin{array}{l}\text { Ley Integral contra la Violencia hacia las Mujeres y de Reformas a la Ley № 641, "Código Penal" (2012) } \\
\text { Available from: http://sgdatabase.unwomen.org/uploads/Ley\%20publicada.pdf }\end{array}$ \\
\hline & Paraguay & $\begin{array}{l}\text { Ley contra la Violencia Doméstica (No. 1600, 2000) } \\
\text { Available from: http://sgdatabase.unwomen.org/uploads/Paraguay\%20-\%20Ley\%201600\%20violencia\%20domestica\%20 } \\
\% 28200 \% 29 . p d f\end{array}$ \\
\hline & Perud,e & $\begin{array}{l}\text { Aprueban el Texto Único Ordenado de la Ley № 26260, Ley de Protección frente a la Violencia Familiar (consolidation of domestic } \\
\text { violence law enacted in } 1993 \text { including reforms enacted through 2008) } \\
\text { Available from: http://sgdatabase.unwomen.org/uploads/Texto\%20Unico.LeyProteccionFamiliar26260\%5B1\%5D.pdf }\end{array}$ \\
\hline
\end{tabular}


TABLE 1. Continued

\begin{tabular}{|c|c|c|}
\hline Region & Country/territory & Name of legislation, year of approval, and source \\
\hline & Puerto Ricod,e & $\begin{array}{l}\text { Ley de Prevención e Intervención con la Violencia Doméstica (1989) } \\
\text { Available from: http://www.lexjuris.com/lexlex/lex89054.htm }\end{array}$ \\
\hline & Saint Lucia & $\begin{array}{l}\text { Domestic Violence (Summary Proceedings) Act, } 1995 \\
\text { Available from: http://www.hsph.harvard.edu/population/domesticviolence/stlucia.dv.95.pdf }\end{array}$ \\
\hline & $\begin{array}{l}\text { Saint Vincent and } \\
\text { the Grenadines }\end{array}$ & $\begin{array}{l}\text { Domestic Violence (Summary Proceedings) Act, } 1995 \\
\text { Available from: http://www.hsph.harvard.edu/population/domesticviolence/SAINTVINCENTANDTHEGRENADINES.htm }\end{array}$ \\
\hline & $\begin{array}{l}\text { Trinidad and } \\
\text { Tobago }\end{array}$ & $\begin{array}{l}\text { Domestic Violence Act, } 1999 \\
\text { Available from: http://sgdatabase.unwomen.org/uploads/Domestic\%20Violence\%20Act\%201999.pdf }\end{array}$ \\
\hline & United States & $\begin{array}{l}\text { Violence Against Women and Department of Justice Reauthorization Act of } 2005 \\
\text { Available from: http://sgdatabase.unwomen.org/uploads/VAWA\%202005.pdf }\end{array}$ \\
\hline & Uruguay & $\begin{array}{l}\text { Ley № 17.514: Violencia Doméstica (2002) } \\
\text { Available from: http://sgdatabase.unwomen.org/uploads/Uruguay\%20-\%20Ley\%201754\%20Violencia\%20Domestica\%20 } \\
\% 282002 \% 29 . p d f\end{array}$ \\
\hline & Venezuelae & $\begin{array}{l}\text { Ley Orgánica sobre el Derecho de las Mujeres a una Vida Libre de Violencia (2007) } \\
\text { Available from: } \\
\text { http://www.ministeriopublico.gob.ve/c/document_library/get_file?p___id=29938\&folderld=14478\&name=DLFE-320.pdf }\end{array}$ \\
\hline \multirow[t]{17}{*}{ Europe } & Albania $^{\dagger}$ & $\begin{array}{l}\text { Law No. } 9669 \text { of } 18.12 .2006 \text { "On Measures Against Violence in Family Relations" (2006) } \\
\text { Available from: http://sgdatabase.unwomen.org/uploads/Albania\%20-\%20Law\%20on\%20Measures\%20against\%20 } \\
\text { Violence\%20in\%20Family\%20Relations\%20\%282006\%29.doc.pdf }\end{array}$ \\
\hline & $\begin{array}{l}\text { Bosnia and } \\
\text { Herzegovina }\end{array}$ & $\begin{array}{l}\text { Law on Protection from Domestic Violence (2005) } \\
\text { Available from: http://www.hsph.harvard.edu/population/domesticviolence/bosnia.domesticviol.05.pdf }\end{array}$ \\
\hline & Bulgaria & $\begin{array}{l}\text { Protection Against Domestic Violence Act (2005) } \\
\text { Available from: http://sgdatabase.unwomen.org/uploads/Protection\%20Against\%20Domestic\%20Violence\%20Act\%202005.pdf }\end{array}$ \\
\hline & Croatiag $^{g}$ & $\begin{array}{l}\text { Act on Protection Against Domestic Violence (2003) } \\
\text { http://sgdatabase.unwomen.org/searchDetail.action?measureld=6015\&baseHREF=country\&baseHREFld=388 }\end{array}$ \\
\hline & Cyprus $^{d}$ & $\begin{array}{l}\text { Violence in the Family (Prevention and Protection of Victims) Laws } 2000 \text { and } 2004 \text { (English translation and consolidation of law enacted } \\
\text { in } 2000 \text { and reforms enacted in 2004) } \\
\text { Available from: http://sgdatabase.unwomen.org/uploads/Cyprus\%20-\%20Violence\%20in\%20the\%20Family\%20 } \\
\% 28 \text { Prevention\%20and\%20Protection\%20of\%20Victims\%29\%20Laws\%202000\%20and\%202004\%20\%28en\%29.pdf }\end{array}$ \\
\hline & Greece $^{\mathrm{g}}$ & $\begin{array}{l}\text { Law 3500/2006 ("For Combating Domestic Violence") and other provisions } \\
\text { http://sgdatabase.unwomen.org/searchDetail.action?measureld=10296\&baseHREF=country\&baseHREFld=563 }\end{array}$ \\
\hline & Ireland ${ }^{\mathrm{d}, \mathrm{e}}$ & $\begin{array}{l}\text { Domestic Violence Act, } 1996 \\
\text { Available from: http://www.irishstatutebook.ie/1996/en/act/pub/0001/print.html } \\
\text { Domestic Violence (Amendment) Act, } 2002 \\
\text { Available from: http://www.irishstatutebook.ie/2002/en/act/pub/0030/print.html }\end{array}$ \\
\hline & Malta & $\begin{array}{l}\text { Domestic Violence Act (2006) } \\
\text { Available from: http://sgdatabase.unwomen.org/uploads/Malta\%20-\%20Act\%20XX\%20on\%20domestic\%20violence\%20 } \\
\text { \%28eng\%29.pdf }\end{array}$ \\
\hline & $\begin{array}{l}\text { Moldova, } \\
\text { Republic of }\end{array}$ & $\begin{array}{l}\text { Law on Preventing and Combating Family Violence (2007) } \\
\text { Available from: http://sgdatabase.unwomen.org/uploads/Law\%20on\%20Preventing\%20and\%20Combating\%20Family\%20 } \\
\text { Violence\%202007.pdf }\end{array}$ \\
\hline & Montenegro $^{e}$ & $\begin{array}{l}\text { Law on Domestic Violence Protection (2010) } \\
\text { Available from: http://legislationline.org/search/runSearch/1/country/57/rows/10/page/2 }\end{array}$ \\
\hline & Poland & $\begin{array}{l}\text { Act of } 29 \text { July } 2005 \text { on Counteraction of Domestic Violence } \\
\text { Available from: http://www.hsph.harvard.edu/population/domesticviolence/domesticviolence.htm }\end{array}$ \\
\hline & Romania & $\begin{array}{l}\text { Law No. } 217 / 2003 \text { on the Preventing and Fighting Against Family Violence (2003) } \\
\text { Available from: } \\
\text { http://sgdatabase.unwomen.org/searchDetail.action?measureld=6269\&baseHREF=country\&baseHREFId=1074 }\end{array}$ \\
\hline & San Marino & $\begin{array}{l}\text { Law No. } 97 \text { of June } 2008 \text { on the Prevention and Elimination of Violence Against Women and Gender Violence } \\
\text { Available from: http://sgdatabase.unwomen.org/searchDetail.action?measureld=10605\&baseHREF=country\&baseHREF } \\
\text { Id=1123 }\end{array}$ \\
\hline & Slovenia & $\begin{array}{l}\text { Family Violence Prevention Act (ZPND, 2008) } \\
\text { Available from: http://sgdatabase.unwomen.org/uploads/Family\%20Violence\%20Prevention\%20Act\%202008.pdf }\end{array}$ \\
\hline & Spain & $\begin{array}{l}\text { Organic Act 1/2004 of } 28 \text { December on Integrated Protection Measures Against Gender Violence } \\
\text { Available from: http://sgdatabase.unwomen.org/uploads/Spain\%20-\%20Organic\%20Act\%20on\%20Integrated\%20 } \\
\text { Measures\%20against\%20Gender-Based\%20Violence\%20\%282004\%29\%20\%28eng\%29.pdf }\end{array}$ \\
\hline & Ukraine $^{e}$ & $\begin{array}{l}\text { Law on Prevention of Domestic Violence (2001) } \\
\text { Available from: http://www.legislationline.org/documents/id/5052 }\end{array}$ \\
\hline & United Kingdom & $\begin{array}{l}\text { Domestic Violence, Crime and Victims Act } 2004 \\
\text { Available from: http://sgdatabase.unwomen.org/uploads/United\%20Kingdom\%20-\%20Domestic\%20Violence\%20Crime\%20 } \\
\text { and\%20Victims\%20Act\%20\%282004\%29.pdf }\end{array}$ \\
\hline
\end{tabular}


TABLE 1. Continued

\begin{tabular}{|c|c|c|}
\hline Region & Country/territory & Name of legislation, year of approval, and source \\
\hline \multirow[t]{22}{*}{ Asia } & Afghanistan & $\begin{array}{l}\text { Law on Elimination of Violence Against Women (EVAW, 2009) } \\
\text { Available from: http://sgdatabase.unwomen.org/uploads/EVAW\%20law\%20-\%202009.pdf }\end{array}$ \\
\hline & Azerbaijan & $\begin{array}{l}\text { Law of the Republic of Azerbaijan on Prevention of Domestic Violence (2010) } \\
\text { Available from: http://sgdatabase.unwomen.org/uploads/Law\%20on\%20Prevention\%20of\%20Domsetic\%20Violence\%202010. } \\
\text { pdf }\end{array}$ \\
\hline & Cambodia & $\begin{array}{l}\text { Law on the Prevention of Domestic Violence and the Protection of Victims (2005) } \\
\text { Available from: http://sgdatabase.unwomen.org/uploads/Law\%20on\%20Prevention\%20of\%20DV\%20and\%20Protection\%20 } \\
\text { of\%20Victims.pdf }\end{array}$ \\
\hline & India & $\begin{array}{l}\text { The Protection of Women from Domestic Violence Act, } 2005 \\
\text { Available from: http://sgdatabase.unwomen.org/uploads/India\%20-\%20Protection\%20of\%20Women\%20from\%20Domestic\%20 } \\
\text { Violence\%20Act.pdf }\end{array}$ \\
\hline & Indonesia & $\begin{array}{l}\text { Law of the Republic of Indonesia Number } 23 \text { of Year } 2004 \text { Regarding Elimination of Violence in Household } \\
\text { Available from: http://sgdatabase.unwomen.org/uploads/Indonesia\%20-\%20Law\%20No\%2023\%202004_Elimination\%20 } \\
\text { on\%20Violence\%20in\%20Household.pdf }\end{array}$ \\
\hline & Israeld $^{d}$ & $\begin{array}{l}\text { Prevention of Family Violence Law, 5751-1991 [as amended by Amendment no. 3, 5748-1997 and Amendment no. 4, 5748-1998] } \\
\text { Available from: http://sgdatabase.unwomen.org/uploads//srael\%20-\%20Prevention\%20of\%20Family\%20Violence\%20Law.pdf }\end{array}$ \\
\hline & Japand $^{d}$ & $\begin{array}{l}\text { Act on the Prevention of Spousal Violence and the Protection of Victims (Revised edition of law } 31 \text { enacted in } 2001 \text { including reforms } \\
\text { enacted through 2007) } \\
\text { Available from: http://www.hsph.harvard.edu/population/domesticviolence/japan.dv.01.pdf }\end{array}$ \\
\hline & Kazakhstan & $\begin{array}{l}\text { Law of the Republic of Kazakhstan on Prevention of Domestic Violence, } 2009 \\
\text { Available from: http://sgdatabase.unwomen.org/uploads/Law\%20on\%20DV\%20-\%202009.pdf }\end{array}$ \\
\hline & $\begin{array}{l}\text { Korea, } \\
\text { Republic of } \mathrm{d}, \mathrm{e}\end{array}$ & $\begin{array}{l}\text { Act on the Prevention of Domestic Violence and Protection, Etc. of Victims Thereof (Revised edition of law } 5487 \text { enacted in } 1997 \\
\text { including reforms enacted through 2006) } \\
\text { Available from: http://sgdatabase.unwomen.org/uploads/Republic\%20of\%20Korea\%20-\%20Act\%20on\%20the\%20 } \\
\text { Prevention\%20of\%20Domestic\%20Violence\%20and\%20Protection,\%20etc,\%20o\%20Victims\%20Thereof.pdf } \\
\text { Prevention of Domestic Violence and Victim Protection Act (1997) } \\
\text { Available from: http://www.wcwonline.org/pdf/lawcompilation/Korea_PreventionAct-1998.pdf }\end{array}$ \\
\hline & Kyrgyzstan & $\begin{array}{l}\text { Law on Social Legal Protection Against Domestic Violence, } 2003 \\
\text { Available from: www.stopvaw.org/uploads/kyrgyz_republic.doc }\end{array}$ \\
\hline & $\begin{array}{l}\text { Lao People's } \\
\text { Democratic } \\
\text { Republic }\end{array}$ & $\begin{array}{l}\text { Law on Development and Protection of Women (2004) } \\
\text { Available from: http://sgdatabase.unwomen.org/uploads/LaoPDR\%20-\%20law\%20and\%20decree\%20on\%20development\%20 } \\
\text { and\%20protection\%20of\%20women_2004-eng.pdf }\end{array}$ \\
\hline & Malaysia & $\begin{array}{l}\text { Domestic Violence Act } 1994 \\
\text { Available from: http://sgdatabase.unwomen.org/searchDetail.action?measureld=10438\&baseHREF=country\&baseHREFId=815 }\end{array}$ \\
\hline & Mongolia & $\begin{array}{l}\text { Law to Combat Domestic Violence (2005) } \\
\text { Available from: http://sgdatabase.unwomen.org/uploads/Law\%20to\%20combat\%20domestic\%20violence\%20-\%202005.pdf }\end{array}$ \\
\hline & Nepal & $\begin{array}{l}\text { Domestic Violence (Offence and Punishment) Act, } 2066 \text { (2009) } \\
\text { Available from: http://sgdatabase.unwomen.org/uploads/DV\%20\%28Crime\%20and\%20Punishment\%29\%20Act\%202009.pdf }\end{array}$ \\
\hline & Pakistan & $\begin{array}{l}\text { Domestic Violence (Prevention and Protection) Act, } 2009 \\
\text { Available from: http://sgdatabase.unwomen.org/uploads/DV\%20Bill\%20-\%202012.pdf }\end{array}$ \\
\hline & Philippines & $\begin{array}{l}\text { Anti-Violence Against Woman and Their Children Act of } 2004 \\
\text { Available from: http://sgdatabase.unwomen.org/uploads/Philippines\%20-\%20anti\%20violence\%20against\%20women\%20 } \\
\text { and\%20children\%20act\%20and\%20implementing\%20rules.pdf }\end{array}$ \\
\hline & Sri Lanka & $\begin{array}{l}\text { Prevention of Domestic Violence Act, No. } 34 \text { of } 2005 \\
\text { Available from: http://sgdatabase.unwomen.org/uploads/Sri\%20Lanka\%20-\%20Prevention\%20of\%20Domestic\%20 } \\
\text { Violence\%20Act\%20\%282005\%29.pdf }\end{array}$ \\
\hline & Taiwane & $\begin{array}{l}\text { Domestic Violence Prevention Act (1998) } \\
\text { Available from: http://law.moj.gov.tw/Eng/news/news_detail.aspx?id=437 }\end{array}$ \\
\hline & Thailand & $\begin{array}{l}\text { Domestic Violence Victim Protection Act, B.E. } 2550 \text { (2007) } \\
\text { Available from: http://sgdatabase.unwomen.org/uploads/DV\%20PTC\%20ACt\%202550\%20-\%202007.pdf }\end{array}$ \\
\hline & Timor-Leste & $\begin{array}{l}\text { Law Against Domestic Violence (No. 7, 2010) } \\
\text { Available from: http://sgdatabase.unwomen.org/uploads/Timor\%20Leste\%20-\%20Law\%20Against\%20Domestic\%20 } \\
\text { Violence\%20Law\%20No.\%207-2010\%20\%28English\%29.pdf }\end{array}$ \\
\hline & Turkey & $\begin{array}{l}\text { Law to Protect Family and Prevent Violence Against Women (2012) } \\
\text { Available from: http://sgdatabase.unwomen.org/searchDetail.action?measureld=54083\&baseHREF=country\&baseHREF } \\
\text { Id=1326 }\end{array}$ \\
\hline & Viet Nam & $\begin{array}{l}\text { Law on Domestic Violence Prevention and Control (2007) } \\
\text { Available from: http://sgdatabase.unwomen.org/uploads/Viet\%20Nam\%20-\%20Law\%20on\%20Domestic\%20Violence\%20 } \\
\text { Prevention\%20and\%20Control\%202007.pdf }\end{array}$ \\
\hline
\end{tabular}


TABLE 1. Continued

\begin{tabular}{|c|c|c|}
\hline Region & Country/territory & Name of legislation, year of approval, and source \\
\hline \multirow[t]{10}{*}{ Africa } & Botswana & $\begin{array}{l}\text { Domestic Violence Act (2008) } \\
\text { Available from: http://sgdatabase.unwomen.org/uploads/DV\%20Act\%202008.pdf }\end{array}$ \\
\hline & Ghana & $\begin{array}{l}\text { Domestic Violence Act (No. 732, 2007) } \\
\text { Available from: http://sgdatabase.unwomen.org/uploads/Ghana\%20-\%20Domestic\%20Violence\%20Act\%20\%282007\%29.pdf }\end{array}$ \\
\hline & Mauritius $^{d}$ & $\begin{array}{l}\text { Protection from Domestic Violence (Amendment) Act } 2007 \\
\text { Available from: http://sgdatabase.unwomen.org/uploads/Mauritius\%20-\%20Protection\%20from\%20Domestic\%20Violence\%20 } \\
\text { \%28Amendment\%29\%20Act\%202007.pdf } \\
\text { Protection from Domestic Violence (Amendment) Act } 2004 \\
\text { Available from: http://sgdatabase.unwomen.org/uploads/Mauritius\%20-\%20Protection\%20from\%20Domestic\%20Violence\%20 } \\
\% 28 \text { Amendment\%29\%20Act\%202004.pdf } \\
\text { Protection from Domestic Violence Act 1997 } \\
\text { Available from: http://www.hsph.harvard.edu/population/domesticviolence/MAURITIU1.htm }\end{array}$ \\
\hline & Mozambique & $\begin{array}{l}\text { Lei sobre a Violencia Doméstica praticada contra a Mulher (No. 29, 2009) } \\
\text { Available from: http://sgdatabase.unwomen.org/uploads/Law\%20on\%20DV\%20-\%202009\%20-\%20in\%20Portuguese.pdf }\end{array}$ \\
\hline & Namibia & $\begin{array}{l}\text { Combating of Domestic Violence Act, } 2003 \text { (No. 4) } \\
\text { Available from: http://sgdatabase.unwomen.org/uploads/Namibia\%20-\%20Domestic\%20Violence\%20Act.pdf }\end{array}$ \\
\hline & Rwanda & $\begin{array}{l}\text { Law on Prevention and Punishment of Gender-Based Violence (No. 59, 2008) } \\
\text { Available from: } \\
\text { http://sgdatabase.unwomen.org/uploads/Rwanda\%20-\%20Law\%20on\%20prevention\%20and\%20punishment\%20of\%20 } \\
\text { GBV\%20\%282008\%29.pdf }\end{array}$ \\
\hline & Seychelles $^{d}$ & $\begin{array}{l}\text { Family Violence (Protection of Victims) Act (revised edition } 2000 \text { ) } \\
\text { Available from: http://sgdatabase.unwomen.org/uploads/Family\%20Violence\%20Protection\%20of\%20Victims\%20Act\%20-\%20 } \\
\text { 2000.pdf }\end{array}$ \\
\hline & Sierra Leone & $\begin{array}{l}\text { Domestic Violence Act, } 2007 \\
\text { Available from: http://sgdatabase.unwomen.org/uploads/Sierra\%20Leone\%20-\%20Domestic\%20Violence\%20Act\%202007.pdf }\end{array}$ \\
\hline & South Africa & $\begin{array}{l}\text { Domestic Violence Act, } 1998 \\
\text { Available from: http://sgdatabase.unwomen.org/uploads/South\%20Africa\%20-\%20Domestic\%20Violence\%20Act\%201998.pdf }\end{array}$ \\
\hline & Zimbabwe & $\begin{array}{l}\text { Domestic Violence Act (No. 24, 2006) } \\
\text { Available from: http://sgdatabase.unwomen.org/uploads/Zimbabwe\%20-\%20Domestic\%20Violence\%20Act\%202007.pdf }\end{array}$ \\
\hline Oceania & New Zealand & $\begin{array}{l}\text { Domestic Violence Act } 1995 \\
\text { Available from: http://sgdatabase.unwomen.org/uploads/Domestic\%20Violence\%20Act\%201995\%20amended\%20as\%20 } \\
\text { of\%201\%20July\%202010.pdf }\end{array}$ \\
\hline
\end{tabular}

\footnotetext{
a Data obtained from the public and private organizations shown in the links above, and from a previous study (30).

${ }^{b}$ Unless noted otherwise, the full legal texts are available from the links above.

$c$ The language of the law titles indicates the language of the legal text available from the links above.

${ }^{d}$ In these countries, family and domestic violence laws and their respective reforms were analyzed simultaneously.

e The legal texts for these laws are available from the public and private organizations shown in the Web pages listed in the table.

f Non-official English translation (as noted within the document).

$g$ The full texts for these laws are not available online; paper copies are available on request from the corresponding author.
}

phasize the role of the judicial system, followed by that of social services and the police. Only 28 countries/territories mentioned the health sector in their VAW legislation. Education and mass media appeared to be the sectors least involved in VAW interventions. References to the various sectors included descriptions of their role in the prevention of VAW and the protection, support, and care of VAW victims as public service entities.

In the Americas, Argentina, Brazil, Colombia, Mexico, Venezuela, and El Salvador all referred to all six sectors in their VAW legislation, and Bolivia, Chile, Nicaragua, Peru, and the United States all referred to the police and judicial systems. Some of the latter five countries also referred to some of the other four sectors. In Europe, Spain was the only country that called for the participation of all six sectors in its VAW legislation. Albania, Bulgaria, Montenegro, the Republic of Moldova, San Marino, and Ukraine all called for the involvement of at least four of the six sectors in their VAW legislation. In Asia, VAW legislation mainly emphasized the role of the police and the judicial system, with the occasional reference to social services, health services, and the education system. The Philippines was the only country/territory that referred to all six sectors in its VAW legislation. Most VAW legislation in Africa only referred to the police and judicial systems (Figure 2).

\section{DISCUSSION}

The results of the content analysis suggest most VAW legislation does not incorporate the key components recommended by PAHO and the UN. This finding seems valid, even though the study inclusion criteria favored relatively recent legislation (versus all VAW legislation from 1984 to 2012), because most of the countries/territories included in the sample (64 out of 80 ) produced or reformed their legislation between January 2000 and June 2012. More than $60 \%$ of the countries/territories formulated their VAW legislation as "domestic violence" law rather than focusing specifically on violence directed against women, and most "domestic 
FIGURE 1. Proportion of countries/territories in each region with violence against women (VAW) legislation analyzed in cross-sectional study measuring levels of commitment to legislative content recommendations from international organizations, by year, January $1984-J u n e ~ 2012^{\mathrm{a}}$

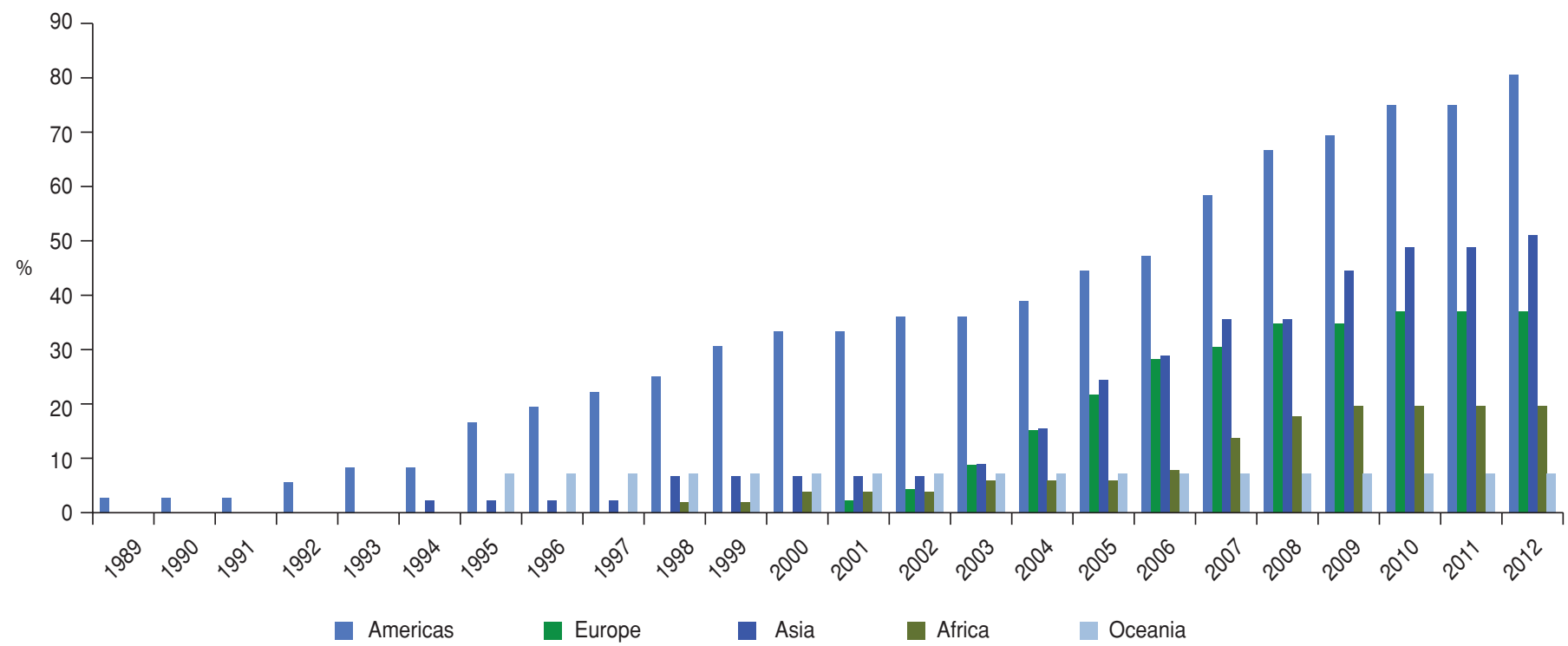

${ }^{a}$ Accessible full-text legislation available in English, Portuguese, or Spanish that includes common terms related to VAW and reference to the participation of at least two of six sectors (education, health, judicial system, mass media, police, and social services) in VAW victim interventions (protection, support, and care).

TABLE 2. Number of countries/territories that identified various populations as beneficiaries of their legislation on violence against women (VAW), by terms used in title, and target beneficiary, January 1984-June 2012

\begin{tabular}{lcccc}
\hline & \multicolumn{4}{c}{ Number of countries/territories with VAW legislation that includes the term } \\
\cline { 2 - 5 } \multicolumn{1}{c}{ Term } & Women & $\begin{array}{c}\text { Women and other } \\
\text { family members }\end{array}$ & The family & Total \\
\cline { 2 - 5 } & 2 & 5 & 44 & 51 \\
\hline "Domestic violence" & 0 & 2 & 6 & 8 \\
"Family violence" & 1 & 0 & 1 & 2 \\
"Gender violence" & 0 & 1 & 0 & 1 \\
"Intra-family violence" & 0 & 4 & 0 & 4 \\
Other & 9 & 5 & 0 & 14 \\
"Violence against women" & 9 & & \\
\hline
\end{tabular}

violence" laws defined the complainant/survivor of the violence as "the family" rather than explicitly mentioning women as the victims. Only 28 countries/territories defined the main forms of VAW ("economic," "physical," "psychological," and "sexual"). Most legislation highlighted the role of the judicial system, social services, and the police in interventions supported by VAW legislation. Most countries/territories in the Americas included key recommended components in their VAW legislation, which may be a result of the legal framework established in that region since the Convention of Belém do Pará (1994) (26).

Most legislation that included the term "violence against women" in the title was enacted in the Americas. Spain and
Rwanda both used the term "gender violence" in the title of their VAW legislation (and were the only countries to do so), but the content of their respective "gender violence" laws differed considerably with regard to other key components. The low frequency of use of the terms "violence against women" and "gender violence" in the titles of the VAW legislation included in the study sample indicates the predominance of "gender-neutral legislation" (legislation that does not specifically identify women as the main victims and survivors of this type of violence $(20,28))$. As shown in many previous studies, legislation labeled as "domestic violence," "intrafamily violence," or "family violence" law has tended to prioritize the stability of the family over the rights of women (20, 21, 27-29).

According to research by $\mathrm{PAHO}$ and the UN, if women are not explicitly recognized as the main complainants and/or survivors of violence in the legal text, the legislation can be manipulated against women $(21,28)$. For example, in some cases, female survivors of violence have been prosecuted for their inability to protect their children from violence (28). Legal analysis of the laws has demonstrated the important distinction between the terms "gender violence" and "domestic violence" in this context. Legislation that uses the latter term generally specifies "the family" as the target beneficiary, whereas legislation that uses the former term generally focuses on the protection of female citizens based on their entitlement to fundamental rights (34). This finding is supported by the current study results, as well as other research $(35,36)$ that demonstrates the scant mention made of women as victims in need of protection-and the notably small number of interventions specifically aimed at victims of VAW - in laws that use the term "domestic violence." In addition, even though the main forms of VAW ("economic," "physical," "psychological," and "sexual") often occur together (sometimes recurrently, within the same relationship), according to the results of the current study, only 28 countries/ 
FIGURE 2. Principal sectors participating in interventions related to violence against women (VAW) based on the promotion of (references to) various sectors in VAW legislation, by country/territory $(n=80)$, January 1984-June 2012

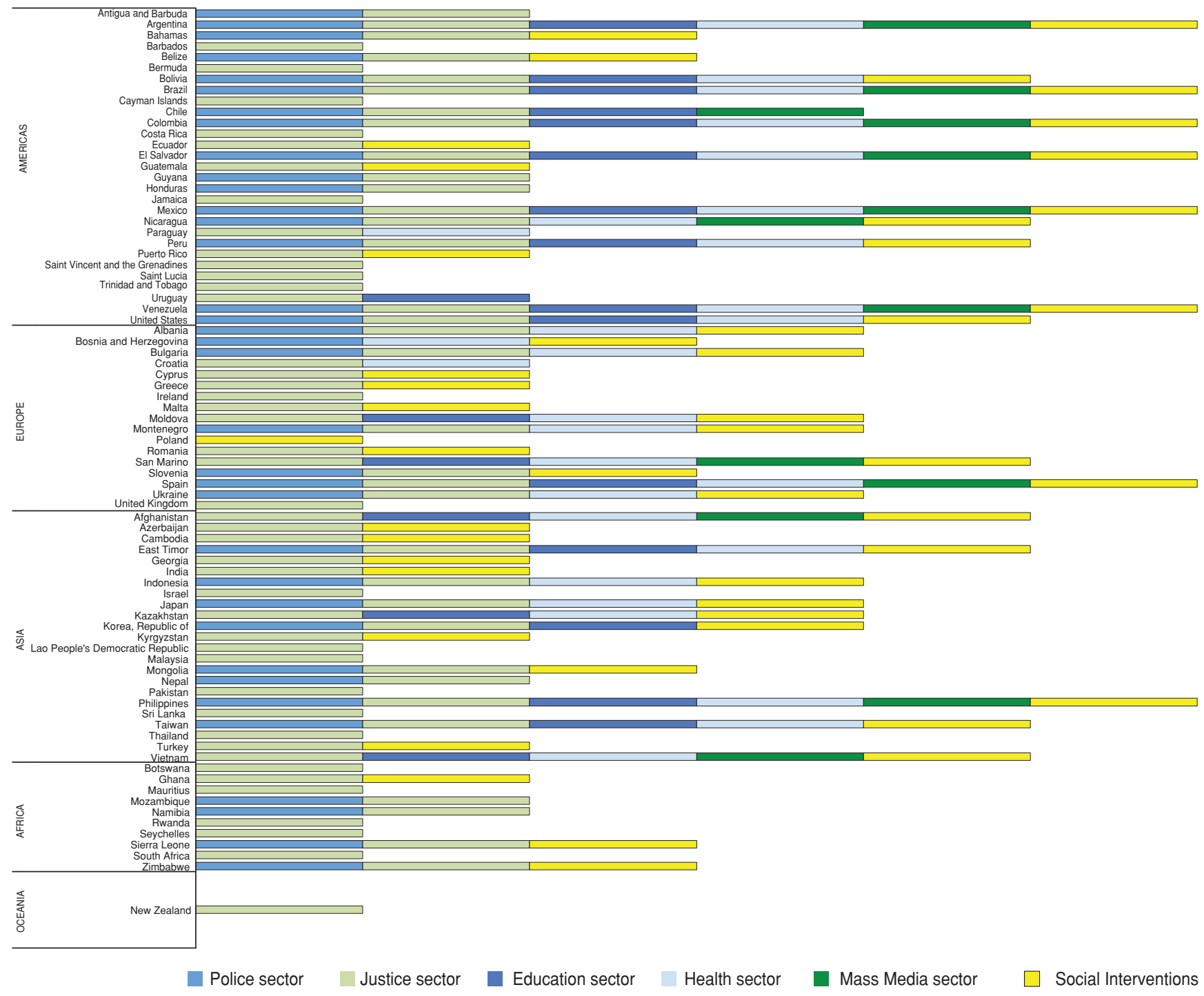

territories included a definition of the four forms of abuse in their VAW legislation. Inclusion and definition of these terms in a legal text have been found to constitute a key requirement for interventions aimed at all of these forms of abuse $(20,21,28)$.

Argentina, El Salvador, Mexico, and Venezuela are notable for their reference to and definition of other forms of VAW (e.g., "institutional," "symbolic," and "media") in their legislation. Guatemala, El Salvador, and Mexico stand out for their inclusion and definition of other, more specific terms for VAW (e.g., "femicide" and "misogyny").
Promotion of the role of various sectors in VAW legislation (e.g., education, health, judicial system, mass media, police, social services) is another legislative component advocated by $\mathrm{PAHO}$ and the UN as a means of facilitating integrated care for VAW victims $(20,21)$. While the current content analysis did not find a high rate of reference to those six sectors, this result should be interpreted with a certain amount of caution, because the cross-sectional study design did not allow for consideration of other national policies that may have included contributions by those sectors to the provision of care for victims of VAW. For example, the United Kingdom recently implemented a new government strategy that resulted in a significant reduction in the number of social services available for victims of VAW. At the same time, however, a new government entity was created to implement a policy designed to improve the housing conditions of women and children made homeless by VAW. In other words, the government transferred responsibility for tackling VAW from the social services sector to a new decision-making entity (23).

The development of "comprehensive legislation" (legislation involving multiple sectors) enables the creation of care 
and support services customized to the needs of female survivors of violence and facilitates the participation of other sectors such as social services and mass media through educational and awarenessraising interventions. Although most VAW legislation still does not promote the participation of most of the six sectors analyzed in this study, current VAW laws are more effective than those enacted 20 years ago. As characterized by some researchers, current VAW legislation can be seen as an efficient social program that prioritizes the care and protection of women who are victims of violence (37).

Public policies influence the determinants of health and are a primary mechanism for carrying out actions that reduce social and economic inequities (e.g., providing equal access for all women in a violent situation to VAW resources and services). Participation of the health sector in victim support and care is strengthened by laws that mandate coordinated interventions with other sectors (38). However, these types of laws may also hinder support and care for victims of VAW. For example, in 1996, Guatemala's Intrafamilial Violence Act (Ley de violencia intrafamiliar) established mandatory reporting by health professionals of all cases of violence. This law became a barrier to efforts to prevent VAW as many health professionals chose to ignore signs of violence to avoid getting involved in criminal cases. The 1996 law was thus superseded in 2008 by the Law Against Femicide and Other Forms of Violence Against Women (Ley contra el Femicidio y otras Formas de Violencia contra la Mujer), which eliminated the reporting requirement.

In Argentina, Brazil, Colombia, El Salvador, Mexico, Spain, and Venezuela, VAW laws call for the participation of the health sector through specific interventions that include the use of intersectoral coordination mechanisms to integrate judicial and social services (38). These types of interventions may play an important role in facilitating access for female victims of abuse to legal and social resources.

\section{Limitations and strengths}

This study had certain limitations that should be considered when interpreting the findings. First, as mentioned in the description of the study inclusion criteria, countries/territories whose VAW legislative text was incomplete or unavailable when the study was carried out were excluded from the study. Therefore, the authors were unable to ascertain if those countries/territories included the key components advocated by PAHO and the UN in their VAW legislation. In addition, as mentioned above, the crosssectional study design did not allow the authors to analyze other national policies (e.g., special social services for immigrant women) that may have entailed contributions to VAW interventions by sectors that were not mentioned in VAW legislation and thus not included in the tabulations of the current study. On the other hand, this study was based on VAW legislation from 80 countries/territories, obtained from three international legal databases that have been used very little to date in research on VAW legislation and policies. Given the challenges in compiling legal documents from countries/territories worldwide, these databases proved to be extremely valuable tools and provided a rich supply of current, reliable, and comprehensive information on a topic with relatively limited research base.

\section{Conclusion and recommendations}

The level of commitment to including the key components recommended for VAW-related legislation by PAHO and the UN is unequally distributed by geographic region and country/ territory. Despite considerable efforts to meet these standards, especially in the Americas, most VAW-related laws do not incorporate these desirable elements and are thus significantly limited in terms of their content and application as well as their ability to provide women with integrated treatment for and protection from violence.

Further modification of VAW-related legislation seems essential to ensure sufficient access of female victims of violence to the justice system and to provide ample protection and social resources to address their needs. Addressing other factors that may affect the policy-making process with regard to gender issues (such as the level of female empowerment in the political sphere), and promoting gender budgeting (conceiving, planning, approving, executing, monitoring, analyzing and auditing budgets in a gender-sensitive way), could help achieve this goal. In developing VAWrelated legislation, policymakers should consider the vital role played by the health services sector in providing access to comprehensive health care.

Acknowledgments. This research was made possible thanks to sponsorship from the Women's Health Observatory (Observatorio de Salud de la Mujer) of the Spanish Ministry of Health, Social Services and Equality (Ministerio de Sanidad, Servicios Sociales e Igualdad). The authors thank Jordi Torrubiano Domínguez and Vicente Clemente of the Universidad de Alicante (Alicante, Spain) for their help with graphic design of the figures.

Conflicts of interest. There are no conflicts of interest related to this article on the part of the authors.

\section{REFERENCES}

1. Krug EG, Dahlberg LL, Mercy JA, Zwi AB, Lozano $R$, editors. World report on violence and health. Geneva: World Health Organization; 2002. Available from: http://whqlibdoc.who.int/ hq/2002/9241545615.pdf Accessed 12 July 2012.
2. Krantz G, García-Moreno C. Violence against women. J Epidemiol Community Health. 2005;59:818-21.

3. Heise LL, Raikes A, Watts $\mathrm{CH}$, Zwi $\mathrm{AB}$. Violence against women: a neglected public health issue in less developed countries. Soc Sci Med. 1994;39(9):1165-79.

4. Krantz G. Violence against women: a global public health issue [editorial]. J Epidemiol Community Health. 2002;56(4):242-3. 
5. García-Moreno C, Jansen HA, Ellsberg M, Heise L, Watts $\mathrm{CH}$; WHO Multi-country Study on Women's Health and Domestic Violence against Women Study Team. Prevalence of intimate partner violence: findings from the WHO multi-country study on women's health and domestic violence. Lancet. 2006;368(9543):1260-9.

6. Campbell JC. Health consequences of intimate partner violence. Lancet. 2002;359(9314): 1331-6.

7. Romito P, Molzan Turan J, De Marchi M. The impact of current and past interpersonal violence on women's mental health. Soc Sci Med. 2005;60(8):1717-27.

8. National Center for Injury Prevention and Control (US). Cost of intimate partner violence against women in the United States. Atlanta: Centers for Disease Control and Prevention; 2003. Available from: www.cdc.gov/ violenceprevention/pdf/IPVBook-a.pdf Accessed 12 July 2012

9. Walby S. The cost of domestic violence. London: UK Department of Trade and Industry Women and Equality Unit; 2004. Available from: www.lancs.ac.uk/fass/sociology/ papers/walby-costdomesticviolence.pdf Accessed 12 July 2012.

10. Browne A, Salomon A, Bassuk SS. The impact of recent partner violence on poor women's capacity to maintain work. Violence Against Women. 1999;5(4):393-426.

11. Reeves C, O'Leary-Kelly A. The effects and costs of intimate partner violence for work organizations. J Interpers Violence. 2007;22(3): $327-44$.

12. Vives-Cases C, Ortiz-Barreda G, Gil-González D. Mapping violence against women laws in the world: an overview of state commitments [editorial]. J Epidemiol Community Health. 2010;64(6):474-5.

13. United Nations Entity for Gender Equality and the Empowerment of Women. Progress of the world's women: in pursuit of justice (2011). New York: UN Women; 2011. Available from: http://progress.unwomen.org/ Accessed 12 July 2012.

14. Office of the United Nations High Commissioner for Human Rights. Vienna Declaration and Programme of Action adopted by the World Conference on Human Rights in Vienna on 25 June 1993. New York: UN; 1993. Available from: www2.ohchr.org/english/ law/vienna.htm Accessed 12 July 2012.

15. United Nations. Report of the Fourth World Conference on Women. New York: UN; 1995. Available from: http://www.un.org/esa/ gopher-data/conf/fwcw/off/a--20.en Accessed 12 July 2012.

16. United Nations General Assembly. Declaration on the Elimination of Discrimination Against Women. New York: UN General Assembly; 1967. (A/RES/2263). Available from: http://www.unhcr.org/refworld/ docid/3b00f05938.html Accessed July 12, 2012.

17. United Nations General Assembly. Convention on the Elimination of All Forms of
Discrimination Against Women. New York: UN General Assembly; 1979. Available from: www.un.org/womenwatch/daw/cedaw/ text/econvention.htm Accessed 12 July 2012.

18. Hawkins D, Humes M. Human rights and domestic violence. Polit Sci Quart. 2002;117(2):231-57.

19. European Women's Lobby. Towards a common European framework to monitor progress in combating violence against women. Brussels: EWL; 2001. Available from: http:// ec.europa.eu/justice_home/daphnetoolkit/ files/projects/2000_046/en_broch_evauatiion_good_pratices_ewl.pdf Āccessed 12 July 2012.

20. United Nations. Handbook for legislation on violence against women. New York: UN; 2010. (ST/ESA/329). Available from: www. un.org/womenwatch/daw/vaw/handbook/Handbook\%20for\%20legislation $\% 20$ on $\% 20$ violence $\% 20$ against $\% 20$ women.pdf Accessed 12 July 2012.

21. Organización Panamericana de la Salud. Modelo de leyes y políticas sobre violencia intrafamiliar contra las mujeres. Washington: OPS; 2004. Available from: www.paho.org/ Spanish/AD/GE/LeyModelo.pdf Accessed 12 July 2012.

22. Organización Panamericana de la Salud. Componentes clave en la formulación de leyes y políticas contra la violencia hacia las mujeres. Documento de discussion. Washington: OPS; 2003. Available from: www.paho. org/Spanish/DPM/GPP/GH/ComponentesClave.pdf Accessed 12 July 2012.

23. Hague G, Malos H. Inter-agency approaches to domestic violence and the role of social services. Br J Soc Work. 1998;28(3):369-86.

24. Pennington-Zoellner, K. Expanding "community" in the community response to intimate partner violence. J Fam Viol. 2009;24(8): 539-45.

25. Organización Panamericana de la Salud. XXXVII Consejo Directivo de la Organización Panamericana de la Salud. Resolución CD 37.R19. Washington: PAHO; 1993. Available from: www.paho.org/Spanish/GOV/CD/ ftcd_37.htm\#R19 Accessed 12 July 2012.

26. Organization of American States. Inter-American Convention on the Prevention, Punishment, and Eradication of Violence Against Women ("Convención of Belem do Pará"). Belem do Pará (Brasil): OEA; 1994. Available from: www.oas.org/en/CIM/docs/Belemdo-Para\%5BEN\%5D.pdf Accessed 12 July 2012.

27. Macaulay F. Judicialising and (de) criminalising domestic violence in Latin America. Social Policy \& Society. 2006;5(1):103-14.

28. United Nations Entity for Gender Equality and the Empowerment of Women. Domestic violence legislation and its implementation: an analysis for ASEAN countries based on international standards and good practices. $2^{\text {nd }}$ ed. Bangkok; UN Women; 2011. Available from: http:/ / cedaw-seasia.org/docs/DomesticViolenceLegislation.pdf Accessed 12 July 2012.
29. United Nations General Assembly. In-depth study on all forms of violence against women: report of the Secretary-General. New York: UN General Assembly; 2006. (A/61/122/ Add.1). Available from: www.unhcr.org/refworld/docid/484e58702.html Accessed 12 July 2012.

30. Ortiz-Barreda G, Vives-Cases C, Gil-González D. Worldwide violence against women legislation: an equity approach. Health Policy. 2011;100(2-3):125-33.

31. Harvard School of Public Health. Annual Review of Population Law [database on the Internet]. Boston: HSPH; c2012. Available from: www.hsph.harvard.edu/population/ annual_review.htm Accessed 12 July 2012.

32. UN Secretary-General's Database on Violence Against Women [Internet]. New York: UN; 2008. Available from: http:// webapps01. un.org/vawdatabase/about.action Accessed 12 July 2012.

33. Advocates for Human Rights. Stop Violence Against Women: domestic violence [Internet]. Available from: www.stopvaw.org/ Domestic_Violence2.html Minneapolis: AHR; c2010-2012. Accessed 12 July 2012.

34. Duran M. Análisis jurídico-feminista de la Ley Orgánica de Medidas de Protección Integral contra la Violencia de Género (artículo 14, una perspectiva de género). Bol Info Anal Jur. 2004;17:4-13.

35. Comisión Interamericana de Mujeres de la Organización de los Estados Americanos. Propuesta de medidas apropiadas para dar seguimiento a la Convención Interamericana para prevenir, sancionar y erradicar la violencia contra la mujer, Convención de Belém do Pará. Washington: OEA; 2004. Available from: www.iidh.ed.cr/comunidades/ derechosmujer/docs/dm interinteres/cimremimiiesp.pdf Accessed 12 July 2012.

36. Larraín S. Curbing domestic violence: two decades of action. Washington: Inter-American Development Bank; 1998. Available from: www.iadb.org/publications/search.cfm?que $\mathrm{ry}=\mathrm{Larra} \% \mathrm{C} 3 \% \mathrm{ADn} \&$ context $=$ Author\&lang $=$ en\&searchLang=all\&searchtype=general Accessed July 12, 2012.

37. Andersen-Clark K, Biddle AK, Martin SL. A cost-benefit analysis of the Violence Against Women Act of 1994. Violence Against Women. 2002;8(4):417-28.

38. Ortiz-Barreda G, Vives-Cases C. Violencia contra las mujeres: el papel del sector salud en la legislación internacional. Gac Sanit. 2012;26(5):483-9.

Manuscript received on 15 November 2011. Revised version accepted for publication on 30 July 2012. 
RESUMEN El estudio se centró en determinar si la legislación sobre la violencia contra la mujer en el mundo contiene los elementos fundamentales que recomiendan la Organización Panamericana de la Salud (OPS) y las Naciones Unidas (NU) para ayudar a fortalecer

\section{Legislación sobre la violencia contra la mujer: panorama de los elementos fundamentales} la prevención de la violencia contra la mujer y brindar mejor protección, apoyo y atención integrados a las víctimas.

Se llevó a cabo una búsqueda sistemática de la legislación sobre la violencia contra la mujer utilizando bases de datos jurídicas internacionales y otras fuentes electrónicas, además de los datos de investigaciones anteriores, y se identificaron 124 países o territorios con algún tipo de legislación sobre la violencia contra la mujer. Se encontraron textos jurídicos completos con legislación de 104 países o territorios. Se descargaron y compilaron aquellos disponibles en inglés, portugués y español, y se sometieron a los criterios de selección (uso de alguno de los términos frecuentes relacionados con la violencia contra la mujer, inclusive la violencia de pareja, y referencia al menos a dos entre seis sectores de servicios [educación, salud, sistema judicial, medios masivos de comunicación, policía y servicios sociales] respecto de las intervenciones relacionadas con la violencia contra la mujer [protección, apoyo y atención]). Se seleccionó una muestra final de 80 países o territorios y se analizó en ella la presencia de los componentes clave recomendados por la OPS y las NU (referencia a la "violencia contra la mujer" en el título; definiciones de diferentes formas de violencia contra la mujer; identificación de las mujeres como beneficiarias; y promoción de [referencia a] la participación de múltiples sectores en las intervenciones relacionadas con la violencia contra la mujer).

Pocos países o territorios identificaron específicamente a las mujeres como beneficiarias de su legislación sobre violencia contra la mujer, incluidos los que denominaban su legislación como ley de "violencia doméstica" $(n=51)$, de los cuales solo dos mencionaban explícitamente a las mujeres como demandantes o supervivientes. Solo 28 países o territorios definieron las formas principales de violencia contra la mujer (económica, física, psicológica y sexual) en su legislación sobre la violencia contra la mujer. La mayoría destacaron la función del sistema judicial, seguida por la función de los servicios sociales y la policía. Solo 28 hicieron mención al sector de la salud.

A pesar de los considerables esfuerzos que se han hecho en todo el mundo para fortalecer la legislación sobre la violencia contra la mujer, la mayoría de las leyes relativas a la violencia contra la mujer no incorporan los componentes clave recomendados. Se han encontrado importantes limitaciones en el contenido legislativo, en su aplicación y en la medida en que se proporcionó a las mujeres protección, apoyo y atención integrados. A la hora de elaborar la nueva legislación sobre la violencia contra la mujer, las instancias normativas deben considerar la función vital de los servicios de salud.

Palabras clave Violencia contra la mujer; violencia doméstica; política de salud; legislación; Américas; Europa (continente); Asia; África; Oceanía. 